\title{
Nuclear Astrophysics with Radioactive Beams
}

\section{J. Vervier}

Institut de Physique Nucléaire, Université Catholique de Louvain, Louvain-la-Neuve, Belgium

\section{Radioactive beams, either at low or high energy, can be very useful for understanding violent events which are encountered in nuclear astrophysics.}

The main aim of the field of nuclear astrophysics is to understand the production of energy and the synthesis of elements in stars and during stellar events. Both processes occur through nuclear reactions [1]. In "quiet" stars like our sun, and in general during non-violent stellar events, the rate of these nuclear reactions is much smaller than the average decay rate of the radioactive nuclei which are often produced by the reactions. In consequence, these radioactive nuclei have ample time to decay before becoming involved in other nuclear reactions, so that mostly reactions between stable nuclei are important.

It is believed that, during "violent" stellar events, examples of which will be given below, the opposite situation prevails, i.e. the average time between succes-

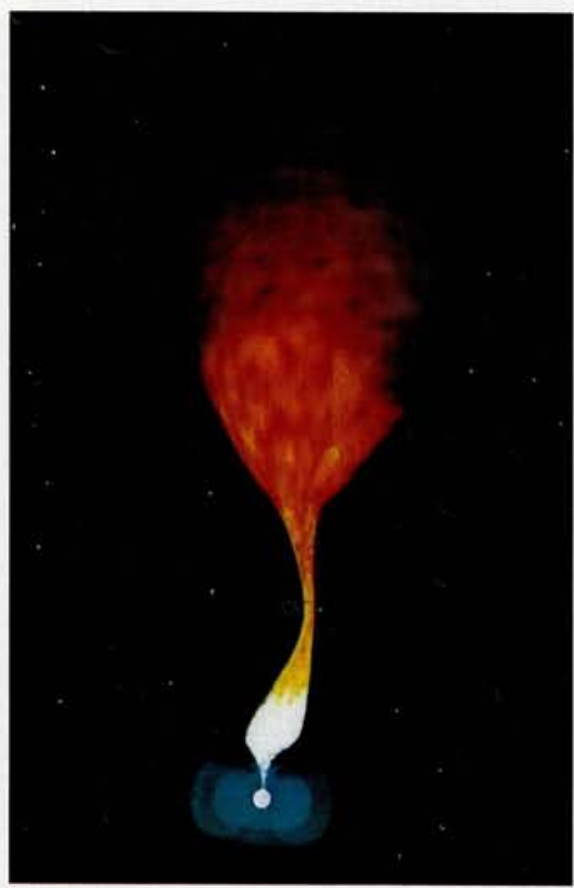

Fig. 1. Schematic model of a novae phenomenon. Matter ejected from a (large) red giant is attracted by à (small) white dwarf, and splashes onto its surface. sive nuclear reactions is much shorter than the average decay time of the radioactive nuclei. These nuclei do not have time to decay before participating in new nuclear reactions, so that reactions in which at least one of the two partners is radioactive (the other one often being a proton or an a $\alpha$-particle) then become very important. It is thus essential, in order to fully understand these violent events, to know the decay properties of the radioactive nuclei produced (half-lives, decay modes etc.) and the cross sections for nuclear reactions in which they are involved. In the present article, we will show that radioactive beams are very useful for the determination of these decay properties and nuclear reaction cross sections.

To measure the cross section of a nuclear reaction in which one of the two partners is radioactive and the other is stable, two methods can be used: the "radioactive target" method, in which the radioactive partner is incorporated in a target which is bombarded by beams of the stable partner; or the "radioactive beam" method, in which a beam of the radioactive partner is produced and used to bombard a target containing the stable partner. It can be shown [1] that, for halflives of the radioactive partner less than about one hour, the radioactive beam method is more efficient, whereas above one hour, the radioactive target method is preferable.

Most of the radioactive nuclei involved in stellar nuclear reactions belong to the first category, so that the development of radioactive beams is necessary for these cross section measurements. Furthermore, radioactive beams are also very useful to determine the decay properties mentioned above, in particular for very exotic nuclei which often play important roles in the violent stellar events. These statements will be illustrated below.
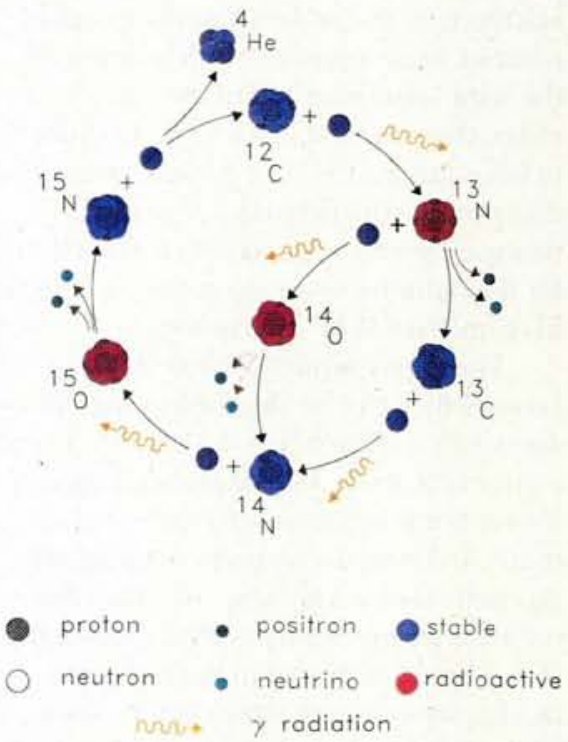

Fig. 2. The cold and hot CNO cycles.

\section{Violent stellar events}

The most spectacular violent stellar events are supernovae, during which the luminosity of a pre-existent star increases suddenly, by factors of $10^{6}$ to $10^{9}$, and later returns to the luminosity of a "normal" star. The so-called type II supernovae correspond to the "death" of a massive star, with a mass between 8 and 100 solar masses, which has undergone the various stages of stellar evolution, from the main sequence phase to the formation of an iron core [1]. At the end of the "life" of such a star, the core collapses as a result of gravity, leading to an implosion followed by a gigantic explosion, the origin of the luminosity increase.

The remnants of such an event include a neutron star, i.e. a very small and very dense object which has many similarities with a giant nucleus, and the supernovae ejecta [2]. During a supernovae event, the temperatures and densities are such that the conditions described above, viz. nuclear reaction rates much higher than average radioactive decay rates, are fulfilled: radioactive nuclei, and the nuclear reactions in which they are involved, play important roles. One of the stellar processes which probably occurs in supernovae is the so-called r-process or rapid-neutron-capture nucleosynthesis [3]. This process involves multiple radiative neutron capture by some target or "seed" nucleus, which are so rapid that the radioactive nuclei created generally do not have time to decay between successive neutron captures.

The r-process is responsible for the 
existence, in the universe, of the so-called $r$-nuclei, i.e. the neutron-rich isotopes of the stable elements, and of the heavy radioactive elements $T h$ and $U$. In order to fully understand the r-process, the decay properties of many very exotic neutron-rich nuclei, and the cross sections for the radiative neutron capture reactions in them, have to be determined.

The most frequent violent stellar events which can be observed in the sky are novae, during which the luminosity of a pre-existent star increases suddenly, as for supernovae, but only by factors of $10^{4}$ to $10^{6}$, and later decreases to the original star luminosity. The "star" in which this occurs is a close binary system (about 50\% of the "stars" observed in the sky are in fact binary systems), which comprises a white dwarf and a red giant. A white dwarf represents the outcome of the hydrogen and helium burning phases of a low mass star like our sun, and has many similarities with a giant atom; a red giant, on the other hand, is a star in the helium burning phase [1]. As shown in Fig. 1, because of the proximity between the two members of the binary system, matter ejected by the red giant is attracted by the white dwarf. After some spiralling around it and formation of a so-called accretion disk, this matter splashes onto the surface of the white dwarf at a very high velocity.

The impact causes a rapid increase in the surface temperature, up to values between 0.1 and $0.5 \times 10^{9} \mathrm{~K}$, leading to the so-called thermonuclear runaway, triggering an explosion at the surface and inducing the luminosity increase [4]. The temperatures and densities which prevail at the surface of the white dwarf during the novae phenomenon are such that some nuclear reaction times become shorter than the decay times of the radioactive nuclei produced. The most important of these reactions is the radiative proton capture on ${ }^{13} \mathrm{~N}$, i.e. ${ }^{13} \mathrm{~N}(\mathrm{p}, \gamma){ }^{14} \mathrm{O}$. The latter reaction distinguishes the so-called "hot CNO cycle", with the following reaction and decay sequence: $\left.\left.{ }^{12} \mathrm{C}(\mathrm{p}, \gamma)\right)^{13} \mathrm{~N}(\mathrm{p}, \gamma)\right)^{14} \mathrm{O}\left(\beta^{+} v_{\mathrm{e}}\right)^{14} \mathrm{~N}(\mathrm{p}, \gamma)^{15} \mathrm{O}\left(\beta^{+} v_{\mathrm{e}}\right)$
${ }^{15} \mathrm{~N}(\mathrm{p}, \alpha){ }^{12} \mathrm{C}$

from the so-called "cold CNO cycle", with sequence:

${ }^{12} \mathrm{C}(\mathrm{p}, \gamma)^{13} \mathrm{~N}\left(\beta^{+} v_{\mathrm{e}}\right)^{13} \mathrm{C}(\mathrm{p}, \gamma)^{14} \mathrm{~N}(\mathrm{p}, \gamma)^{15} \mathrm{O}\left(\beta^{+} v_{\mathrm{e}}\right)$ ${ }^{15} \mathrm{~N}(\mathrm{p}, \alpha){ }^{12} \mathrm{C}$

The cold CNO cycle is an important source of energy in the main sequence stars, i.e. stars at the hydrogen burning stage, and it contributes a few percent to the energy generation by our sun. The hot $\mathrm{CNO}$ cycle is a crucial element in the thermonuclear runaway during the novae events: its characteristic time is that of the longest half-life of the radioactive nuclei it contains, i.e. ${ }^{15} \mathrm{O}\left(\mathrm{T}_{1 / 2}=2 \mathrm{~min}\right.$.), which is five times smaller than for the cold $\mathrm{CNO}$ cycle which includes the $\beta^{+}$-decay of ${ }^{13} \mathrm{~N}$ $\left(\mathrm{T}_{1 / 2}=10 \mathrm{~min}\right.$.). Consequently, the rate of energy production by the hot $\mathrm{CNO}$ cycle is about five times higher than by the cold $\mathrm{CNO}$ cycle. The two cycles are schematically represented in Fig. 2 . In order to determine the stellar conditions under which the cold and the hot CNO cycles respectively dominate, it is crucial to know the cross section of the ${ }^{13} \mathrm{~N}(\mathrm{p}, \gamma)^{14} \mathrm{O}$ reaction, which competes with the $\beta^{+}$decay of ${ }^{13} \mathrm{~N}$ as shown in (1) and (2) above.

A third type of violent stellar event is represented by X-ray bursts, during which "objects" emitting X-rays undergo a sudden increase in their X-ray luminosity followed by a fading to the preburst level of $\mathrm{X}$-radiation; these bursts are repeated by the same object at irregular intervals. The origin of these events is believed to be similar to that of novae. X-ray bursters are also close binary systems, comprising a neutron star and a companion star, for example a red giant. Matter emitted by the companion star is also accreted at the surface of the neutron star, where it induces uncontrolled thermonuclear reactions leading to the sudden increase of X-ray emission [5]. The temperatures and densities at the surface of the neutron star during an X-ray burst are also high enough to allow nuclear reactions between radioactive nuclei to occur. They are however higher than during the novae phenomenon (the temperatures ranging from 0.7 to $1.5 \cdot 10^{9} \mathrm{~K}$ ), so that "more difficult" reactions, i.e. with lower cross sections, may occur. Of the latter reactions, the sequence:

${ }^{15} \mathrm{O}(\alpha, \gamma){ }^{19} \mathrm{Ne}(\mathrm{p}, \gamma){ }^{20} \mathrm{Na}$

leads to a so-called "escape" from the $\mathrm{CNO}$ cycles, because it transforms CNO elements into ${ }^{20} \mathrm{Na}$, which cannot be converted back into lighter nuclei. From there on the so-called rp-process, or rapid-protoncapture nucleosynthesis, may occur. This process is somewhat analogous to the rprocess mentioned above since it involves multiple radiative proton capture reactions on proton-rich nuclei, and it may lead to the nucleosynthesis of medium mass nuclei up to the $\mathrm{Kr}$ region. In order to fully understand the escape from the CNO cycles and the rp-process, the decay properties of the very exotic proton-rich nuclei up to mass 80 , and the cross sections for nuclear reactions involving them, such as those of sequence (3), have to be determined.

These are some examples of violent events encountered in nuclear astrophysics. Others, like type I supernovae, primordial nucleosynthesis and possibly $\gamma$ ray bursts, will not be discussed further.

\section{Production of radioactive beams for nuclear astrophysics}

Two general methods are used to produce radioactive beams for nuclear astrophysics: the Isotope Separator On Line (ISOL) or two-accelerator method, and the fragmentation method. In the ISOL method large quantities of radioactive nuclei are generated by bombarding a thick (primary) target with high-intensity primary stable beams, protons, light or heavy ions, produced by a first accelerator (or with the neutrons of a nuclear reactor). Many different radioactive nuclei are thereby produced, depending on the type and energy of the projectiles, which are stopped in the target. These nuclei are extracted from the target as atoms or molecules, transformed into ions by a suitable ion source, massseparated by an Isotope Separator On Line, and subsequently accelerated by a second accelerator.

The radioactive secondary beams thereby obtained are projected onto a (secondary) target, where nuclear reactions of astrophysical interest can be observed, or are collected by a catcher, where nuclear spectroscopic experiments can be performed. The energies of these secondary beams range from a few tens of $\mathrm{keV}$ (absolute) to a few tens of $\mathrm{MeV}$ per nucleon, depending on the subsequent accelerating scheme. In the lower limit, i.e. near Isotope Separators On Line, spectroscopic experiments on very exotic nuclei, protonor neutron-rich, can be performed whose significance for nuclear astrophysics has been emphasized above. In the upper limit, and especially between 0.2 and $1 \mathrm{MeV}$ per nucleon, one can directly measure the cross sections for nuclear reactions of astrophysical interest involving radioactive nuclei. Examples of such experiments carried out in European laboratories will be described below.

In the fragmentation method, a high energy (from several tens to several hundreds of $\mathrm{MeV}$ per nucleon) primary heavy- 
ion stable beam is focused onto a thin (primary) target where it is fragmented into many nuclei, most of which are radioactive. These fragments are mostly emitted in the forward direction, with about the same velocity as the primary beam. The desired radioactive beams, with energies comparable to the primary beam, are separated from the latter and from the other fragments by suitable methods, involving magnetic and electric fields, wedge-shaped energy degraders, velocity filters, time-of-flight measurements, and so on. These secondary radioactive beams are then projected onto a (secondary) target to study nuclear reactions of astrophysical interest, or are collected by a suitable catcher to investigate their spectroscopic properties. The nuclear reactions thereby studied sometimes allow the indirect determination of the cross sections for other nuclear reactions characteristic of nuclear astrophysics. The exotic nuclei whose spectroscopic properties can be investigated in this way are often close to or even at the proton and neutron drip lines, and their significance for the astrophysical rp- and r-processes has been indicated above. Examples of these measurements performed in European laboratories will be given below.

\section{Direct and indirect cross section measurements}

The ${ }^{13} \mathrm{~N}(\mathrm{p}, \gamma)^{14} \mathrm{O}$ reaction, whose importance in the novae phenomenon has been stressed above, has been investigated by direct and indirect methods. In the former case, $\mathrm{a}^{13} \mathrm{~N}$ beam with an energy of 8.2 $\mathrm{MeV}$ was produced by the ISOL method using the radioactive beam facility at Louvain-la-Neuve, Belgium [6], represented in Fig. 3. The first (production) acceler- ator is the $30-\mathrm{MeV}$ cyclotron CYCLONE 30 , able to produce beam intensities up to 500 $\mu \mathrm{A}$; the (primary) target is a ${ }^{13} \mathrm{C}$ powder which yields ${ }^{13} \mathrm{~N}$ nuclei through the ${ }^{13} \mathrm{C}(\mathrm{p}, \mathrm{n}){ }^{13} \mathrm{~N}$ reaction. The ion source is of the Electron Cyclotron Resonance (ECR) type. The second (subsequent) accelerator is the cyclotron CYCLONE, suitably modified to yield low-energy ( $0.63 \mathrm{MeV}$ per nucleon) ${ }^{13} \mathrm{~N}$ beams, which also plays the role of mass separator. The resulting ${ }^{13} \mathrm{~N}$ beam was incident on a $\left(\mathrm{CH}_{2}\right)_{n}$ polyethylene target, and the capture $\gamma$-rays resulting from the ${ }^{13} \mathrm{~N}(\mathrm{p}, \gamma)^{14} \mathrm{O}$ reaction were observed by Ge detectors. Using this method, the cross section for the latter reaction was measured in the energy range of the first resonance at $0.586 \mathrm{MeV}$, which corresponds to the first excited level of ${ }^{14} \mathrm{O}$ at $5.152 \mathrm{MeV}$ [7]. Complementary experiments on this reaction, dealing with ${ }^{13} \mathrm{~N}(\mathrm{p}, \mathrm{p}){ }^{13} \mathrm{~N}$ elastic scattering and the ${ }^{13} \mathrm{~N}(\mathrm{~d}, \mathrm{n})^{14} \mathrm{O}$ reaction, were also carried out [8].

The same reaction has been investigated using an indirect method based on the following ideas. The principle of detailed balance, which is based on the time-reversal invariance of the strong interaction, allows the cross sections of the two inverse reactions ${ }^{13} \mathrm{~N}(\mathrm{p}, \gamma)^{14} \mathrm{O}$ (radiative capture) and ${ }^{14} \mathrm{O}(\gamma, \mathrm{p})^{13} \mathrm{~N}$ (photodisintegration) to be uniquely related. The cross section for the latter reaction can in turn be measured, not with real photons, but with the virtual photons from the Coulomb field of a heavy nucleus: this is the so-called Coulomb dissociation method. At the LISE facility of the GANIL laboratory at Caen, France [9], represented in Fig. 4, a $70-\mathrm{MeV}$ per nucleon ${ }^{14} \mathrm{O}$ beam has been produced by the fragmentation method described above. A 96-MeV per nucleon ${ }^{16} \mathrm{O}$ primary beam was fragmented

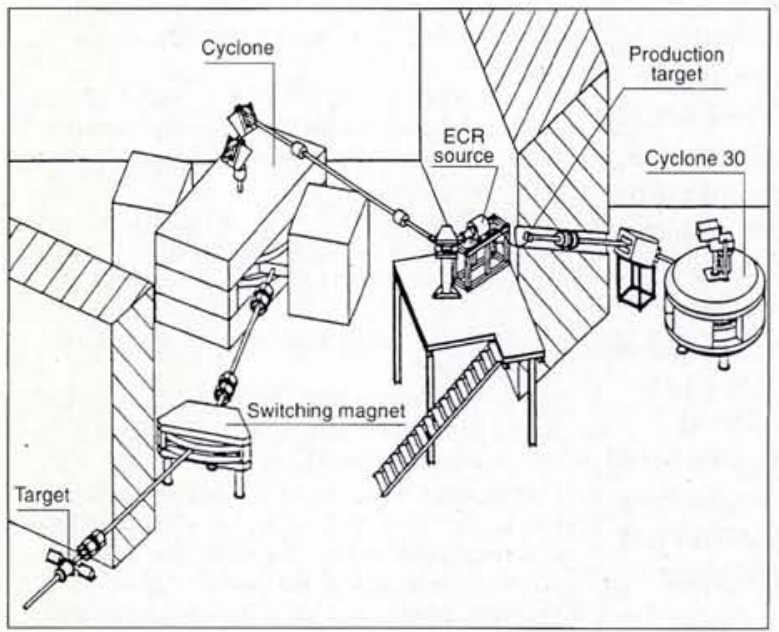

Fig. 3. The radioactive beam facility at Louvain-la-Neuve, Belgium.

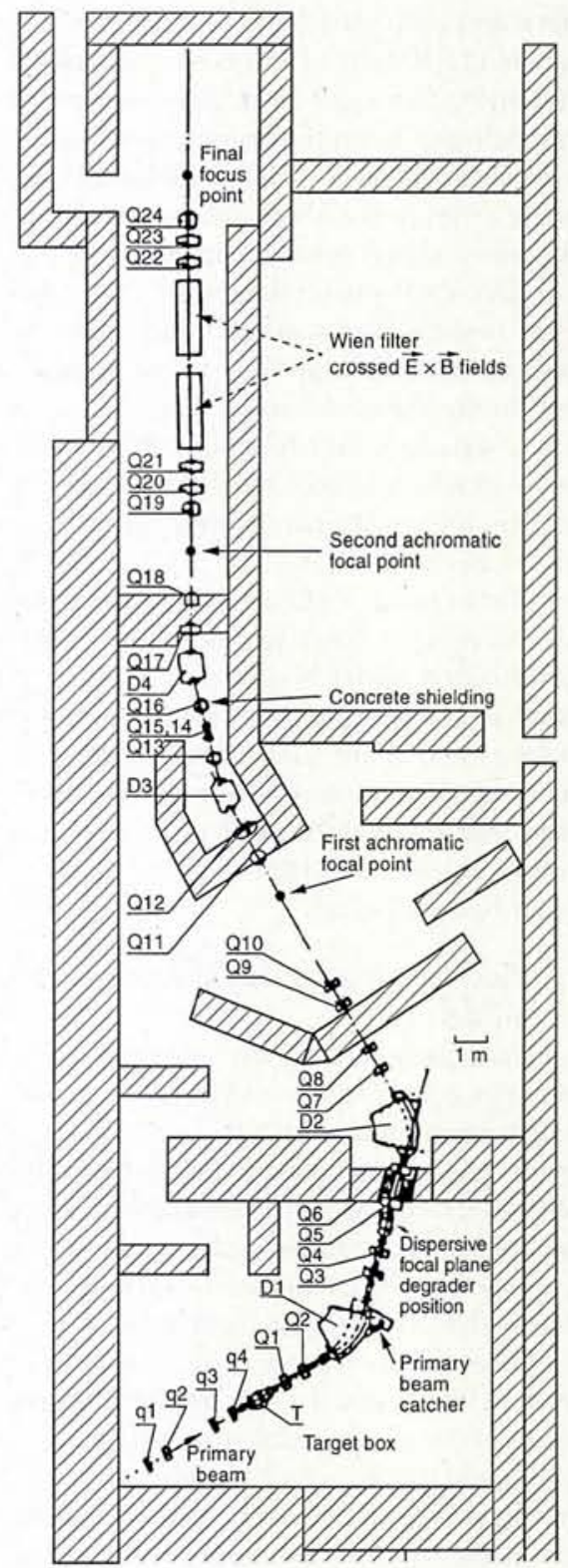

Fig. 4. The LISE facility at GANIL, Caen, France.

by a thin carbon (primary) target, and the ${ }^{14} \mathrm{O}$ fragments were selected by two dipole magnets, an intermediate energy degrader and several quadrupole magnets. The resulting ${ }^{14} \mathrm{O}$ beam was projected onto a ${ }^{208} \mathrm{~Pb}$ (secondary) target, and the ${ }^{13} \mathrm{~N}$ nuclei and protons resulting from the ${ }^{14} \mathrm{O}(\gamma, \mathrm{p})^{13} \mathrm{~N}$ reaction were detected and identified. In this way the cross section for the ${ }^{13} \mathrm{~N}(\mathrm{p}, \gamma)^{14} \mathrm{O}$ reaction was determined in the energy range of the $0.586-\mathrm{MeV}$ resonance mentioned above [10].

The results from the two experiments, as well as those from other indirect measurements dealing with the ${ }^{13} \mathrm{~N}(\mathrm{p}, \gamma)^{14} \mathrm{O}$ reaction, agree with each other within their respective uncertainties. They have enabled the astrophysical conditions, tem- 
peratures and densities to be determined, under which the cold and hot CNO cycles [(2) and (1) above and Fig. 2] dominate respectively. From the conditions which are believed to prevail at the surface of the white dwarf in the novae phenomenon described above, one can conclude that the hot $\mathrm{CNO}$ cycle predominates the cold $\mathrm{CNO}$ cycle in these events, and is thus mainly responsible for the surface explosion causing the luminosity increase in novae.

These data have therefore put the "scenario" worked out by the astrophysicists to explain the novae phenomenon on firm experimental ground.

Other reactions of astrophysical interest involving radioactive nuclei have been investigated, either by direct methods (such as the ${ }^{19} \mathrm{Ne}(\mathrm{p}, \gamma)^{20} \mathrm{Na}$ reaction mentioned in (3) above [11]), or by indirect methods. The results of these experiments have shed light on the mechanisms responsible for the violent stellar events described above [12].

\section{Nuclear spectroscopy of exotic nuclei}

Isotope Separators On Line produce very low energy ( $a$ few tens of $\mathrm{keV}$ ) radioactive beams, which include rather exotic nuclei. As an example, the ISOLDE facility at CERN, Geneva, Switzerland, allows the separation and study of such nuclei as are produced by the irradiation of various targets with 1- $\mathrm{GeV}$ protons from the CERN PS-booster [13]. In particular, it has been used to investigate the spectroscopic properties of the neutron-rich nuclei ${ }_{29}^{79} \mathrm{Cu}_{50}$, ${ }_{30}^{80} \mathrm{Zn}_{50}$ and ${ }_{48}^{130} \mathrm{Cd}_{82}$, which are very far from stability and whose neutron numbers are magic, viz. they are more stable than their neighbours. These nuclei play important roles in the r-process mentioned above, and the results of the experiments dealing with them indicate the duration of this rapid process, i.e. that it is longer than 5 to $10 \mathrm{~s}$ [14].

The fragmentation technique also produces radioactive beams of very exotic nuclei, which are either proton or neutron rich, and whose spectroscopic properties can also be investigated. Sometimes the simple fact that a nucleus is stable or unstable with respect to the emission of a nucleon is valuable evidence for nuclear astrophysics. As a first example, the fragments of a $73-\mathrm{MeV}$ per nucleon ${ }^{78} \mathrm{Kr}$ beam were analysed and identified using the LISE facility at GANIL; the conclusion was that ${ }^{69} \mathrm{Br}$ is particle unstable. This nucleus is in the sequence of the rp-process described above, where it could be pro- duced by the (rapid) proton capture on ${ }^{68} \mathrm{Se}$ whose half-life is $1.6 \mathrm{~min}$. Its particle instability shows that the rp-process is terminated, or at least considerably slowed down, at ${ }^{68}$ Se whose half-life is much longer than the expected rp-process time scale [15]. As a second example, a high-energy (750 $\mathrm{MeV}$ per nucleon) radioactive beam of the very long half-life nucleus ${ }^{238} \mathrm{U}$ was fissioned by beryllium or lead targets. The fission fragments were separated and identified by the Fragment Recoil Separator at the GSI, Darmstadt, Germany [16], using techniques developed for the fragmentation method described above. In this way, more than 50 very neutron-rich new isotopes have been identified, including the magic neutron nucleus ${ }_{28}^{78} \mathrm{Ni}_{50}$, which intervene in the r-process mentioned earlier [17].

The spectroscopy of many other exotic nuclei of interest to nuclear astrophysics has been investigated using similar techniques [12].

\section{Perspectives}

The field of nuclear astrophysics with radioactive beams is a relatively new one which is likely to expand in the next few years. One reason will be the improvement of the existing radioactive beam facilities and the construction of new ones, especially in Europe. For the ISOL method, the performance of the Louvain-laNeuve facility will be improved in 1997 by commissioning the new post-accelerating cyclotron CYCLONE44, with an expected intensity increase of about one order of magnitude. The REX-ISOLDE programme at CERN [18] and the EXCYT project in Catania, Italy [19], which are both expected to work in 1998-1999, will deliver radioactive beams in the energy region suitable for direct cross section measurements on nuclear reactions of astrophysical interest.

The SPIRAL facility at GANIL [20], to be commissioned in 1998-1999, will span a higher energy region (between 2 and 25 $\mathrm{MeV}$ per nucleon) which will yield indirect information on these cross sections and permit the nuclear spectroscopy of exotic nuclei to be studied. The PIAFE project near the nuclear reactor of the LaueLangevin Institute at Grenoble, France [21], is aimed at the prolific production of very neutron-rich nuclei. All these programmes based on the ISOL method should yield radioactive beams which will be more intense and/or more exotic than those presently available, and should widen the field of study of astrophysically interesting nuclear reactions.
Concerning the fragmentation method, intensity upgrades are scheduled at the two currently running European facilities, at GANIL and GSI. This will allow spectroscopic studies to be performed on very exotic nuclei, i.e. not just the proof of their particle stability, but also measurement of their masses, half-lives, delayed-particle emission probabilities etc., in mass regions further from stability than presently achievable.

All these planned experiments will also benefit from the development of new instrumentation: recoil mass separators, multiple $\gamma$-ray detectors, multiple chargedparticle and neutron detector arrays and more.

The experiments already performed in nuclear astrophysics with radioactive beams, and those which will be possible in the next few years, implement the prophetic words of the late W.A. Fowler pronounced on the occasion of his Nobel Lecture at Stockholm in 1983 : "It is my view that continued development and application of radioactive ion beam techniques could bring the most exciting results in laboratory nuclear astrophysics in the next decade" [22].

\section{References}

[1] C.E. Rolfs and W.S. Rodney, Cauldrons in the Cosmos, The University of Chicago Press, Chicago and London (1988).

[2] H.A. Bethe and G.E. Brown, Scientific American 252, 40 (May 1985).

[3] J.J. Cowan, F.K. Thielemann and J.W. Truran, Physics Reports 208, 267 (1991).

[4] J.W. Truran, Ann. Rev. Nucl. Part. Sci. 34, 53 (1984).

[5] R.E. Tamm, Ann. Rev. Nucl.Part. Sci. 35, 1 (1985). [6] D. Darquennes et al., Phys. Rev. C42, R804 (1990).

[7] P. Decrock et al., Phys. Rev. Lett. 67, 808 (1991). [8] P. Decrock et al., Phys. Lett. B304, 50 (1993). [9] A.C. Mueller and R. Anne, Nucl. Instr. Meth. Phys. Res. B56/57, 559 (1991).

[10] J. Kiener et al., Nucl, Phys. A552, 66 (1993).

[11] R. Page et al., Phys. Rev. Lett. 73, 3066 (1994);

C. Michotte et al, Phys. Lett. B381, 402 (1996).

[12] J. Vervier, Progr. Part. Nucl. Phys. 37, 435

(1996).

[13]A.C. Mueller, Proc. Third Int. Conf. Radioactive Nuclear Beams, Editions Frontières, p. 1 (1993).

[14] K.L. Kratz et al., Zeits. Phys. A325, 489 (1986); A340, 419 (1991).

[15] B. Blank et al., Phys. Rev. Lett. 74, 4611 (1995).

[16] Report GSI-95-05 (unpublished).

[17] Ch. Engelmann et al., Zeits. Phys. A352, 351 (1995).

[18] G. Correia et al., Report CERN/ISC 94-25 (unpublished).

[19] D. Vincigueira et al., Proc. Int. Conf. Exotic Nuclei and Atomic Masses ENAM'95, Editions

Frontières, p. 837 (1995).

[20] Report GANIL R-94-02 (unpublished). [21] J.L. Belmont et al., Proc. Int. Workshop Physics and Techniques of Secondary Nuclear Beams, Editions Frontières, P. 407 (1992).

[22] W.A. Fowler, Rev. Mod. Phys. 56, 149 (1984). 